\title{
An in vivo study of hypoxia-inducible factor- $1 \alpha$ signaling in ginsenoside Rg1-mediated brain repair after hypoxia/ischemia brain injury
}

\author{
Binzhi Tang ${ }^{1,2}$, Dejian Wang ${ }^{3}$, Maojun Li ${ }^{1,2}$, Qing Wu ${ }^{1,2}$, Qian Yang ${ }^{1,2}$, Wei Shi ${ }^{1,2}$ and Changhui Chen ${ }^{1,2}$
}

\begin{abstract}
BACKGROUND: Hypoxia/ischemia (HI) brain injury is a common central nervous system insult in newborns. Studies have demonstrated bioactivity of ginsenoside Rg1 in increasing neural viability and promoting angiogenesis. However, there are few reports on roles of $\mathrm{Rg} 1$ in brain repair of neonatal $\mathrm{HI}$, and the mechanisms involved are unclear.

METHODS: a neonatal HI model was established by a modified Rice-Vannucci model (RVM) and pups received ginsenoside Rg1 or monosialotetrahexosyl ganglioside (GM1) treatment. Neurological function and pathologic damage of rats were evaluated. Cellular apoptosis was detected with Terminal deoxynucleotidyl transferase dUTP nick end labeling (TUNEL) assay. Immunohistochemistry for von willebrand factor (vwf) was used to label micro vessels. Expression levels of hypoxia-inducible factor-1 $\alpha(H I F-1 \alpha)$, vascular endothelial growth factor (VEGF), and cleaved caspase 3 (CC3) were detected by western blot.
\end{abstract}

RESULTS: Both Rg1 and GM1 reduced neurological impairment and pathologic damage after $\mathrm{HI}$ by enhancing neural survival. Rg1, but not GM1, could also facilitate angiogenesis after $\mathrm{HI}$. These pharmacological effects of Rg1 may be attributed to regulation of expression level of VEGF and CC3 and HIF$1 \alpha$ signaling pathway was involved.

CONCLUSION: Rg1 plays a neuroprotective role in brain repair following neonatal $\mathrm{HI}$, and $\mathrm{HIF-1} \mathrm{a}$ is a potential target for therapeutic intervention in neonates with HI brain injury.

$\mathbf{H}$ ypoxia-ischemia (HI) brain injury is one of the major perinatal causes of neurological dysfunctions in newborns. A series of investigations indicated that brain repair following HI was associated with enhanced cell survival and angiogenesis in and around the injured regions $(1,2)$. In recent years, many beneficial effects of $\mathrm{Rg} 1$, one of the major active ingredients of Ginseng (a traditional Chinese herb), on the nervous and cardiovascular systems have been reported $(3,4)$. Both in vivo (5) and in vitro (6) experiments revealed that neuronal cell apoptosis induced by HI stress could be reversed by Rg1, further studies suggested that mechanisms underlying antiapoptotic effect of $\mathrm{Rg} 1$ involved inhibiting the mitochondrial apoptotic pathway, activation of neurotrophic factors, and inactivation of proapoptotic proteins such as caspase- 3 (refs. $(7,8))$. Besides, researches have demonstrated Rg1 exerts angiogenic effects in a vascular endothelial growth factor (VEGF)-dependent manner $(9,10)$. However, the signal transduction pathways through which Rg1 modifies expression or activity of such factors are not clearly understood.

We have previously demonstrated hypoxia-inducible factor$1 \alpha$ (HIF-1 $\alpha$ ), the regulatory subunit of HIF-1, is a nuclear factor that modulates many HI-related processes including energy metabolism, vasculogenesis, apoptosis, and migration, by controlling transcription of its downstream factors such as VEGF (7,10-14). On the basis of these findings, we hypothesized that in neonatal HI brain injury, ginsenoside Rg1 mediates activation and strengthen of HIF- $1 \alpha$, which may be beneficial for neuroprotection after HI. To test this hypothesis, we set up a neonatal $\mathrm{HI}$ model using postnatal day 10 rats by a modified Rice-Vannucci model (RVM). We examined effects of Rg1 on neuronal apoptosis and angiogenesis after HI and compared with those of monosialotetrahexosyl ganglioside (GM1), a medicine used clinically in many neurological diseases including neonatal HI brain injury, and investigated expression variations of HIF- $1 \alpha$ and its downstream factors related to apoptosis and angiogenesis such as CC3 and VEGF after HI with or without Rg1 treatment.

\section{RESULTS}

Rg1 Reduced Neurological Impairment and Pathologic Damage After $\mathrm{HI}$ and Had No Neurotoxicity in Developing Rat Brain Longa scoring was used to evaluate neuroethology of neonatal Sprague-Dawley (SD) rats as shown in Figure 1. Abnormality of neural functions, including activity suppression, movement disorder on the right side became manifest after $\mathrm{HI}(P<0.05$ at $4,24,72 \mathrm{~h}$ and $7 \mathrm{~d}$ ) (Figure 1a,b) (data of 4 and $24 \mathrm{~h}$ not shown), and a relief of neurological impairment was observed in both Rg1- $(P<0.05$ at $72 \mathrm{~h}$ and $7 \mathrm{~d}$; Figure 1a,b) and GM1-treated group $(P<0.05$ at $7 \mathrm{~d}$; Figure $1 \mathrm{~b})$. There was no significant

'Department of Pediatrics, Sichuan Academy of Medical Sciences \& Sichuan Provincial People's Hospital, Chengdu, China; ${ }^{2}$ Department of Pediatrics, Clinical College of University of Electronic Science and Technology of China, Chengdu, China; ${ }^{3}$ Department of Pharmacology, Chengdu University of Traditional Chinese Medicine, Chengdu, China. Correspondence: Changhui Chen (chen966888@aliyun.com)

Received 8 May 2016; accepted 13 July 2016; advance online publication 5 October 2016. doi:10.1038/pr.2016.178 
a

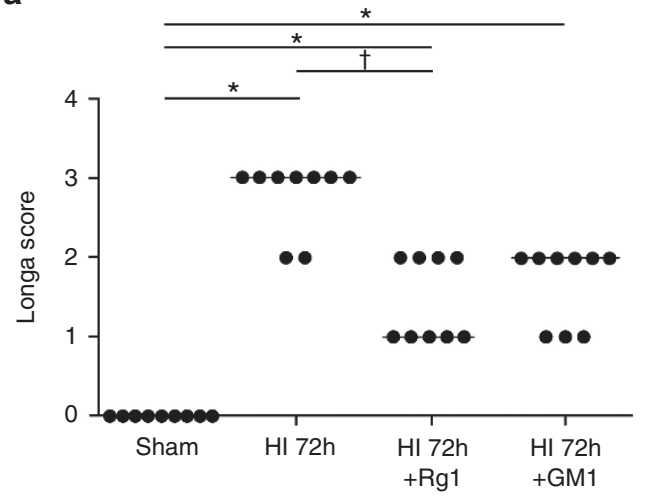

b

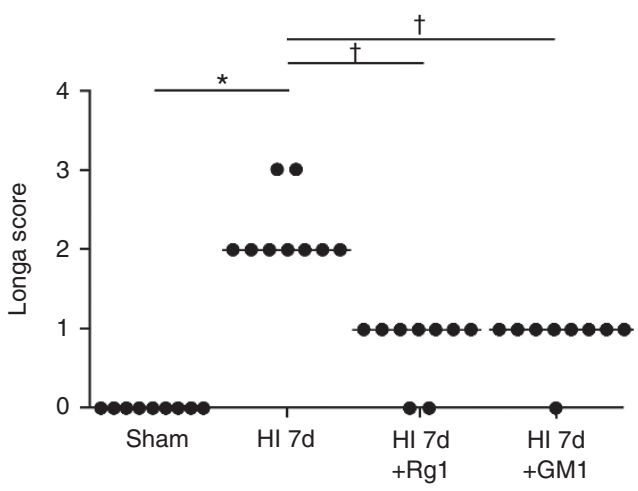

Figure 1. Comparison of neuroethology evaluated by Longa scoring ( $n=9$ for each time point at each group). Significant increase of neurological impairment was observed in animals subjected to $\mathrm{HI}$ at both $72 \mathrm{~h}(\mathbf{a})$ and $7 \mathrm{~d}(\mathbf{b})$ after $\mathrm{HI}(* P<0.05$ compared with sham control). Intraperitoneal injection of Rg1 $(40 \mathrm{mg} / \mathrm{kg})$ significantly reduced neural dysfunctions at both $72 \mathrm{~h}(\mathbf{a})$ and $7 \mathrm{~d}(\mathbf{b})$ while $\mathrm{GM} 1(40 \mathrm{mg} / \mathrm{kg})$ showed its neuroprotective effect at $7 \mathrm{~d}$ (b) after neonatal $\mathrm{HI}(+P<0.05$ compared with $\mathrm{HI}$ group). A notable recovery of neuroethology was found at $7 \mathrm{~d}$ after brain $\mathrm{HI}$ insult in either Rg1 or GM1 treated pups $(P>0.05$ compared with sham control).

difference between Rg1- and GM1-treated group $(P>0.05$ at $4,24,72 \mathrm{~h}$ and $7 \mathrm{~d}$ ) (4 and $24 \mathrm{~h}$, data not shown).

Seventy-two hours after HI, gross specimens of the right hemisphere in sham group showed no obvious pathologic changes (Figure 2a). In contrast, a cortical infarction with geographic borders could be clearly seen in pups in HI group (black arrow, Figure 2b) while minor changes such as edema or hyperemia appeared in pups received Rg1 (Figure 2c) or GM1 treatment (Figure 2d).

HE staining revealed evidence of neuronal damage such as disordered arrangement of neurons and neural edema in the cortex and hippocampus at $72 \mathrm{~h}$ after HI (black arrow, Figure 2f), while there was no obvious damage in sham controls (Figure 2e). Less severe pathologic changes caused by HI exposure were observed in both Rg1 group (Figure 2g) and GM1 group (Figure $2 \mathrm{~h}$ ). Similarly, $72 \mathrm{~h}$ after HI, there were only a few apoptotic cells in the sham controls (Figure 2i), meanwhile significantly more apoptotic ones in the HI brains (black arrow, Figure 2j), apoptotic cells were drastically decreased by Rg1 (black arrow, Figure 2k) and moderately decreased by GM1 (black arrow, Figure 2l) as demonstrated by TUNEL labeling. Neuronal apoptotic index (AI) at different time point after HI was demonstrated in Figure $2 \mathrm{~m}$. Shamoperated rats received continuous intraperitoneal injection of Rg1 or GM1 for $7 \mathrm{~d}$ were also included to explore adverse effects of drugs on neuronal viability in the immature brain. AI of sham $(3.03 \pm 0.74 \%)$, sham $+\operatorname{Rg} 1 \quad(2.96 \pm 0.80 \%)$, and sham+GM1 $(2.94 \pm 0.73 \%)$ measured by TUNEL labeling were all at a low level after $7 \mathrm{~d}$ of drug administration, and there were no significant differences between these three groups $(P>0.05$, figure not shown).

\section{Rg1 Facilitated Angiogenesis After HI}

To investigate the effects of Rg1 and GM1 on angiogenesis in neonatal rats' brain following HI, we used immunohistochemistry (IHC) for von willebrand factor (vwf) to label micro vessels (black arrows, Figure 3a-d). IHC staining showed the number of vwf-positive cells at $72 \mathrm{~h}(24.000 \pm 3.847)$ and $7 \mathrm{~d}(25.000 \pm 4.050)$ in HI group was significantly increased compared to that in sham group $(19.000 \pm 2.608$ at $72 \mathrm{~h}$ and $19.833 \pm 3.060$ at $7 \mathrm{~d}$ respectively, $P<0.05)$, and the number of vwf-positive cells at $7 \mathrm{~d}$ in $\mathrm{Rg} 1$ group $(30.167 \pm 5.115)$ was significantly increased compared to that in HI group at the same time point $(25.000 \pm 4.050, P<0.05)$. However, results from our study suggested angiogenetic effect of GM1 was inferior to that of $\mathrm{Rg} 1$. There was no significant difference between $\mathrm{HI}+\mathrm{GM} 1$ group and HI group (Figure 3e).

\section{Rg1 Strengthened HI Induced HIF-1 $\alpha$ Signaling}

To investigate the mechanism of Rg1-mediated neuroprotection following HI, we collected the right brain tissues from pups received Rg1 or normal saline (NS) treatment at different time points after HI for IHC (Figure 4) and western blot analysis (Figure 5). Distribution and localization of HIF- $1 \alpha$ in P10 rat brains were determined by IHC. We found that expression of HIF- $1 \alpha$ was mainly distributed in the ischemic cortex and hippocampus, and HIF- $1 \alpha$ protein was mainly located in the nucleus and cytoplasm of neurocytes by IHC staining (Figure 4-c), a similar distribution of expression was also found in VEGF (Figure 4d-f) and CC3(Figure 4-i). Therefore in this study, ischemic cortex and hippocampus of right hemisphere were selected for observation of HE staining, IHC staining, TUNEL labeling, and subsequent western blot analysis.

To quantify HIF-1 $\alpha$, VEGF, and CC3 expression, we measured HIF-1 $\alpha$, VEGF, and CC3 protein expression using western blot analysis. As demonstrated in Figure 5, we found that one band of $110 \mathrm{kDa}$, corresponding to HIF- $1 \alpha$ protein (Figure 5a) was obviously induced at $4 \mathrm{~h}(0.207 \pm 0.022), 24 \mathrm{~h}$ $(0.196 \pm 0.025)$, and $72 \mathrm{~h}(0.180 \pm 0.020)$ after HI, compared to sham controls $(0.115 \pm 0.019, P<0.05)$, and then declined at $7 \mathrm{~d}$ $(0.140 \pm 0.018, P>0.05)$. Compared with that of HI group, the expression of HIF- $1 \alpha$ in HI+Rg1 group was further upregulated and maintained a high level for a longer time $(0.396 \pm 0.049$ at $4 \mathrm{~h}, 0.357 \pm 0.043$ at $24 \mathrm{~h}, 0.339 \pm 0.040$ at $72 \mathrm{~h}$, and $0.276 \pm 0.036$ 


\section{Articles | Tang et al.}
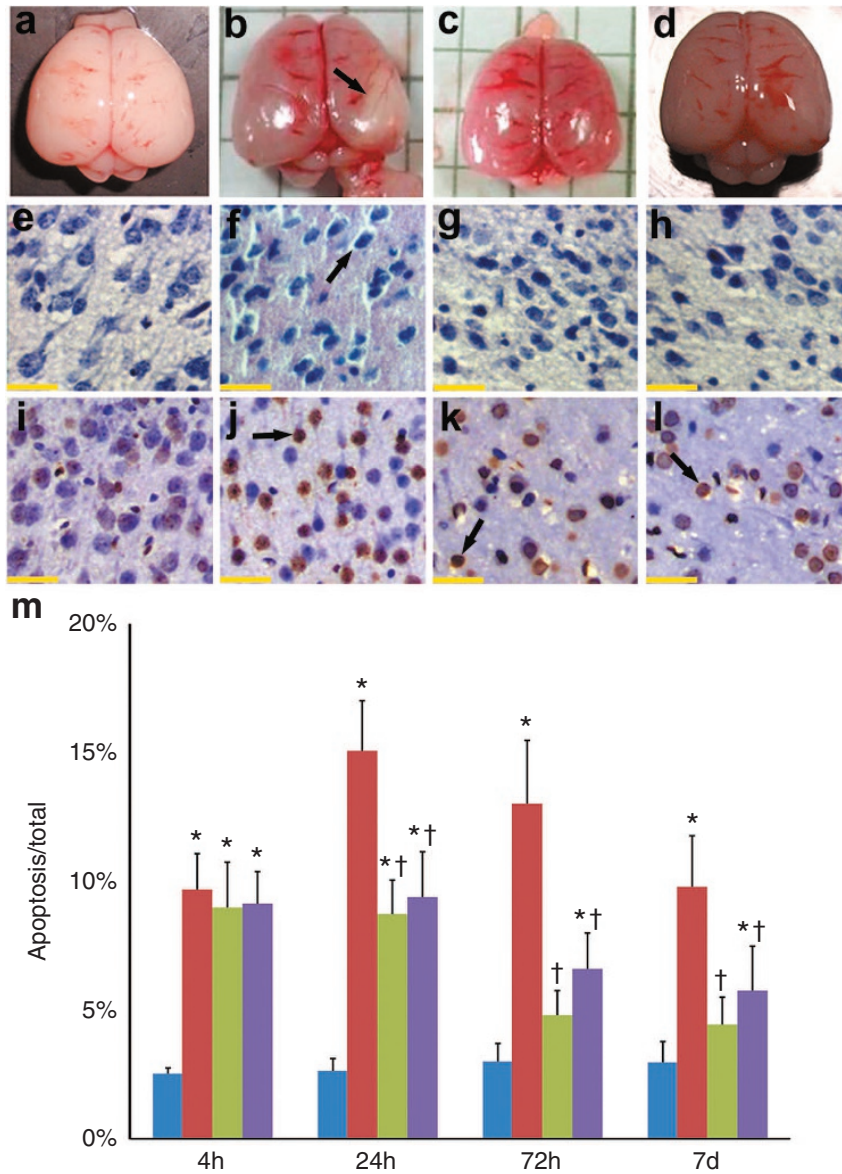

Figure 2. Pathologic changes and neuronal apoptosis at $72 \mathrm{~h}$ after $\mathrm{HI}$ detected by HE (e-h) or TUNEL (i-l). At $72 \mathrm{~h}$ following $\mathrm{HI}$, (a) gross specimens of the right hemisphere in sham controls showed a normal appearance, (b) meanwhile a cortical infarction appeared in HI group (black arrow) and no obvious infarction could be observed in pups received $\mathrm{Rg} 1$ (c) or GM1 (d) treatment. (e) Neural cells with normal morphology and regular arrangement were seen in in sham controls, (f) while disordered arrangement of neurons and neural edema at $72 \mathrm{~h}$ after $\mathrm{HI}$ was revealed by HE (black arrow), meanwhile a mild disordered arrangement of neurons or neural edema was observed in pups received $\operatorname{Rg} 1$ (g) or GM1 (h). (i-I) Three fields in the cortex and hippocampus of each slice were randomly selected at $\times 400$ magnifications for cell count. As revealed by TUNEL, apoptotic ones were characterized by yellow or brown particles confined within the nucleus (black arrows). (i) There were only a few apoptotic cells in sham group and (j) rose significantly at $72 \mathrm{~h}$ after neonatal $\mathrm{HI}$, and were reduced by of Rg1 (k) or GM1 (I). (m) Neuronal apoptotic index (Al) was significantly increased at $4 \mathrm{~h}$ (2.8-folds), $24 \mathrm{~h}$ (4.7-folds), $72 \mathrm{~h}$ (3.3-folds), and $7 \mathrm{~d}$ (2.3-folds) after $\mathrm{HI}$ when compared with sham controls. Al in $\mathrm{HI}+\mathrm{Rg} 1$ group was significantly lower at $24 \mathrm{~h}, 72 \mathrm{~h}$, and $7 \mathrm{~d}$ after $\mathrm{HI}(\mathrm{HI}+$ $\mathrm{Rg} 1$ ) when compared with that in $\mathrm{HI}$ group, with decreasing rate of $42.1 \%$ at $24 \mathrm{~h}, 63.1 \%$ at $72 \mathrm{~h}$, and $54.8 \%$ at $7 \mathrm{~d}$ respectively. A similar decreased $\mathrm{Al}$ level was also observed in GM1-treated (HI + GM1) group, that was $37.6 \%$ lower than that in $\mathrm{HI}$ group at $24 \mathrm{~h}, 49.3 \%$ lower at $72 \mathrm{~h}$, and $41.2 \%$ lower at $7 \mathrm{~d}$ after $\mathrm{HI}$. At $72 \mathrm{~h}$ and $7 \mathrm{~d}$ after $\mathrm{HI}$, there was no significant difference between neuronal $\mathrm{Al}$ in $\mathrm{HI}+\mathrm{Rg} 1$ group $(4.8 \%$ at $72 \mathrm{~h}$ and $4.4 \%$ at $7 \mathrm{~d}$ ) and that in sham controls ( $3.1 \%$ at $72 \mathrm{~h}$ and $3.0 \%$ at $7 \mathrm{~d}$ ). However neuronal $\mathrm{Al}$ in $\mathrm{HI}+\mathrm{GM} 1$ group $(6.6 \%$ at $72 \mathrm{~h}$ and $5.8 \%$ at $7 \mathrm{~d}$ ) was still distinctive from that in sham controls. Histogram: blue fill $=$ sham, red fill $=\mathrm{HI}$, green fill $=$ $\mathrm{HI}+\mathrm{Rg} 1$, violet fill $=\mathrm{HI}+\mathrm{GM} 1 .{ }^{*} P<0.05$ compared with sham control; $+P<0.05$ compared with $\mathrm{HI}$ group. $n=6$ for each time point at each group. Scale bar $20 \mu \mathrm{m}$.
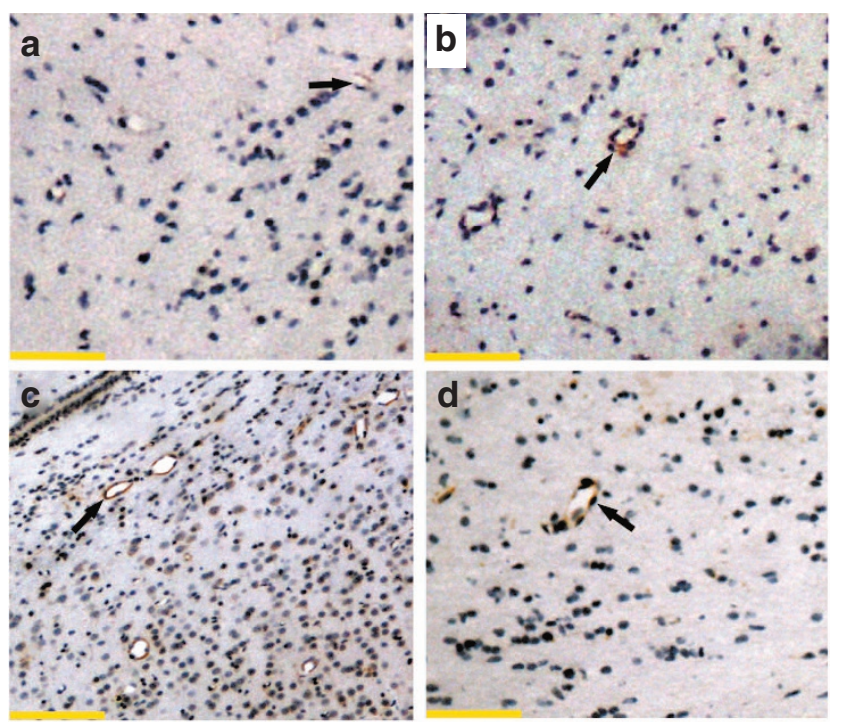

e

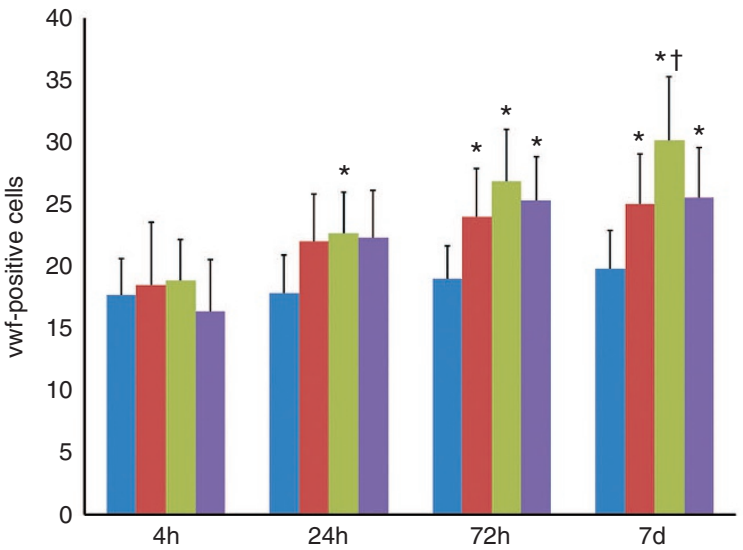

Figure 3. Angiogenetic effects of different treatment detected by immunohistochemistry for von willebrand factor (vwf). (a-d) Three fields in and around the cortex and hippocampus of each slice were chosen randomly at $\times 100$ magnifications to count vwf-positive cells. The micro vessels were characterized by yellow or brown vwf-positive cells circled around (black arrows). Seven days after $\mathrm{HI}$, (a) there were a few micro vessels in sham controls and (b) more micro vessels formed in HI group. (c) HI-induced angiogenesis was further improved by Rg1, (d) meanwhile GM1 showed no significant angiogenetic effect. (e) The number of vwf-positive cells rose significantly at $72 \mathrm{~h}$ (1.27-folds) and $7 \mathrm{~d}$ (1.26-folds) after $\mathrm{HI}$ compared with sham controls. At $7 \mathrm{~d}$, a significant increasing (1.22-folds) of micro vessels was observed in right hemisphere of rats received intraperitoneal injection of $\mathrm{Rg} 1(\mathrm{HI}+\mathrm{Rg} 1)$ compared with those received NS injection (HI). However, there was no significant difference between number of vwf-positive cells in $\mathrm{HI}+\mathrm{GM} 1$ group and that in $\mathrm{HI}$ group at any time point. Histogram: blue fill $=$ sham, red fill $=\mathrm{HI}$, green fill $=\mathrm{HI}+\mathrm{Rg} 1$, violet fill $=\mathrm{HI}+\mathrm{GM} 1 .{ }^{*} P<0.05$ compared with sham control; $+P<0.05$ compared with $\mathrm{HI}$ group. $n=6$ for each time point at each group. Scale bar $100 \mu \mathrm{m}$.

at $7 \mathrm{~d}$ respectively, $P<0.05$ ) (Figure $5 \mathrm{~b}$ ). Meanwhile, one band of $42 \mathrm{kDa}$, corresponding to VEGF protein (Figure 5a), demonstrated a similar expression trend, which was obviously induced by $\mathrm{HI}$ stress at $4 \mathrm{~h}(0.658 \pm 0.079), 24 \mathrm{~h}(0.684 \pm 0.070)$, and $72 \mathrm{~h}$ $(0.503 \pm 0.079)$ compared to sham controls $(0.393 \pm 0.054$, $P<0.05)$ and then declined at $7 \mathrm{~d}(0.463 \pm 0.076)$. Similar 

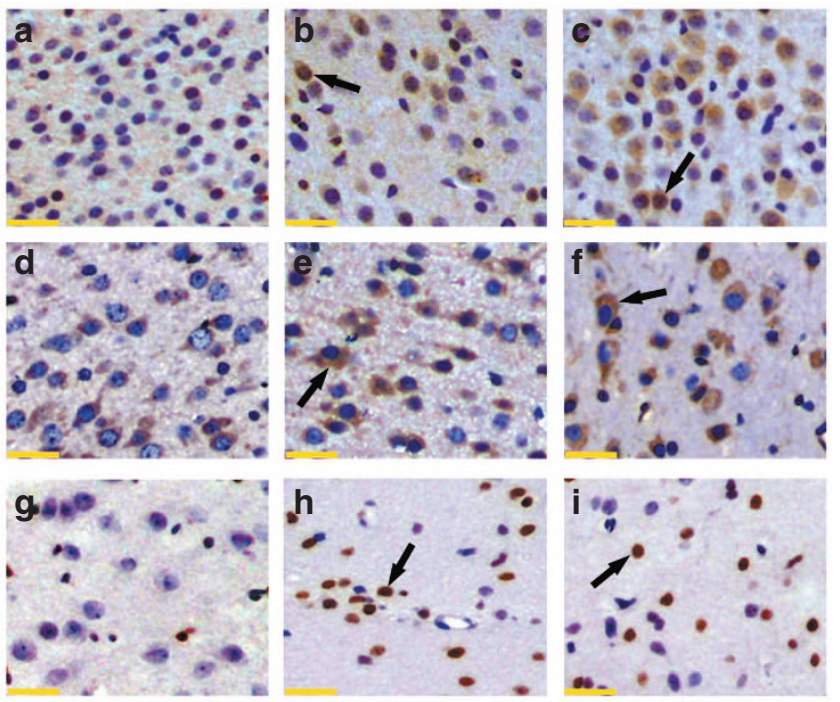

Figure 4. Observation of in situ expression of HIF-1 $\alpha$, VEGF, and CC3 by immunohistochemistry (IHC) staining. Specimens from three different groups (sham, $\mathrm{HI}$ and $\mathrm{HI}+\mathrm{Rg} 1$ group) at $72 \mathrm{~h}$ after $\mathrm{HI}$ were taken for $\mathrm{IHC}$ examples. Three fields in the cortex and hippocampus of each slice were chosen randomly at $\times 400$ magnifications. (a-c) HIF-1 $\alpha$ protein was characterized by yellow or brown particles located in the nucleus and cytoplasm of neurocytes (black arrows). (d-f) VEGF protein was characterized by yellow or brown particles located in the cytoplasm of neurocytes (black arrows). (g-i) CC3 protein was characterized by yellow or brown particles located in the nucleus of neurocytes (black arrows). It could be roughly seen from IHC staining that (a) expression of HIF-1 $\alpha$, (d) VEGF and (g) CC3 were all at a low level in sham group. (b) HIF-1 $\alpha$, (e) VEGF and (h) CC3 were all induced by $\mathrm{HI}$ stress, and (c) expression of HIF-1 $\alpha$ and (f) VEGF were further raised by $\mathrm{Rg} 1$. (i) In contrast, expression of CC3 was decreased by Rg1 treatment. Scale bar $20 \mu \mathrm{m}$.

to HIF-1 $\alpha$, VEGF protein expression was also significantly enhanced by $\operatorname{Rg} 1(0.707 \pm 0.089$ at $4 \mathrm{~h}, 0.824 \pm 0.104$ at $24 \mathrm{~h}$, $0.788 \pm 0.102$ at $72 \mathrm{~h}$, and $0.728 \pm 0.080$ at 7 d respectively, $P<0.05)$, compare to that in $\mathrm{HI}$ group (Figure $5 \mathrm{c}$ ). However, two bands of 17 and $19 \mathrm{kDa}$, corresponding to CC3 protein (Figure 5a), which were also upregulated after HI $(0.480 \pm 0.066$ at $4 \mathrm{~h}$, $0.521 \pm 0.095$ at $24 \mathrm{~h}, 0.466 \pm 0.050$ at $72 \mathrm{~h}$, and $0.328 \pm 0.037$ at $7 \mathrm{~d}$, respectively) compared to sham controls $(0.105 \pm 0.019$, $P<0.05)$, showed a distinct variation of expression from that of HIF-1 $\alpha$ /VEGF. HI-stimulated CC3 expression was significantly downregulated by $\operatorname{Rg} 1(0.332 \pm 0.044$ at $4 \mathrm{~h}, 0.207 \pm 0.032$ at $24 \mathrm{~h}, 0.163 \pm 0.029$ at $72 \mathrm{~h}$, and $0.136 \pm 0.021$ at $7 \mathrm{~d}$ respectively, $P<0.05)$ (Figure 5d).

\section{DISCUSSION}

Ginseng, the root and rhizome of Panax ginseng C A Meyer (a Chinese herb), has been used as a tonic remedy in Chinese traditional medicine for over 2,000 y. In recent years, many beneficial effects of $\mathrm{Rg} 1$, one of the major active ingredients of ginseng, on the nervous and cardiovascular systems have been reported, including increasing neuronal cell viability (15) and promotion of angiogenesis (16). However, there were few researches to determine pharmacological effects of $\mathrm{Rg} 1$ on the central nervous system (CNS) in neonatal HI brain injury and the signaling involved.
In this study, we used a modified RVM to establish an animal HI model in the P10 SD rats. Characteristics of HI insult, such as abnormality of neural function evaluated by Longa scoring (17), cortical infarction, pathologic changes like neural edema, increased neuronal apoptosis, were observed after surgery, suggesting a successful animal model mimicking term human neonates with HI brain injury.

In both in vitro $(6)$ and in vivo $(3,7,18)$ studies, $\mathrm{Rg} 1$ has been shown to protect neurons from HI injury. In this research, we studied the neuroprotection of $\mathrm{Rg} 1$ in neonatal HI brain injury by comparing with GM1, because both Rg1 and GM1 were regarded as neurotrophic factors and mechanisms underlying neuroprotective effects of GM1 may be associated with its antioxidant activities, its ability to modulate neuroplasticity, its potential in promoting neurogenesis, or inhibition of neuronal death $(19,20)$, which were similar to those of $\operatorname{Rg} 1$ (12). We found that neurological impairment and pathologic damage became manifest after neonatal $\mathrm{HI}$ and these manifestations were largely attenuated by both Rg1 and GM1. Using TUNEL labeling, we found neuronal apoptosis rose significantly after neonatal HI, and this apoptotic effect induced by HI could be reduced by both $\mathrm{Rg} 1$ and GM1. These results suggested a neuroprotective role of both Rg1 and GM1 following HI, by protecting against HI-induced neuronal apoptosis. Besides, no obvious increasing of cell death was found in sham-operated pups subjected to $7 \mathrm{~d}$ of $\mathrm{Rg} 1$ administration, suggesting its safety in clinical use.

Regeneration and functional recovery of new blood vessels are vital for brain repair after neonatal HI. Von willebrand factor (vwf) has been well recognized and acknowledged as a specific and sensitive marker for endothelial cells of blood vessels (21), thus in this study we used IHC for vwf to label micro vessels in rats' brain. Results showed Rg1, but not GM1, facilitated angiogenesis after neonatal HI, suggesting multi-potential and advantages of $\mathrm{Rg} 1$ in protecting neonatal CNS against $\mathrm{HI}$.

HIF- $1 \alpha$ has been realized to be involved in modulating many pathologic processes related to $\mathrm{HI}$ insults, including $\mathrm{HI}$ brain injury, by regulating transcription of crucial genes involved in cell viability and vasculogenesis (13), which are also regulated by $\operatorname{Rg} 1(7,10,12)$. Thus, HIF- $1 \alpha$ and its related signaling are likely to be involved in $\operatorname{Rg} 1$ mediated anti-apoptosis and angiogenesis effects after neonatal HI brain injury.

Increase of mitochondrial membrane permeability during $\mathrm{HI}$ activates caspase-3 to form cleaved caspase-3 (CC3), which triggers DNA damage and neuronal cell apoptosis. Previous investigations have suggested a negative correlation between HIF- $1 \alpha$ and CC3 expression in a HI brain injury model of neonatal rats $(7,22)$. Additionally, increasing evidence shows that besides angiogenesis, VEGF contributes to brain protection by inhibiting activation of CC3 (23) and thus enhancing survival and reducing cell apoptosis of vascular endothelial cells, as well as neurons (23-26). We therefore explored the effects of Rg1 on HIF-1 $\alpha$, VEGF and CC3 expression to find out how Rg1 regulates neuronal apoptosis following neonatal HI. In agreement with previous reports $(7,10,22)$, expression of HIF-1 $\alpha$, VEGF and CC3 were all upregulated after HI. Western 


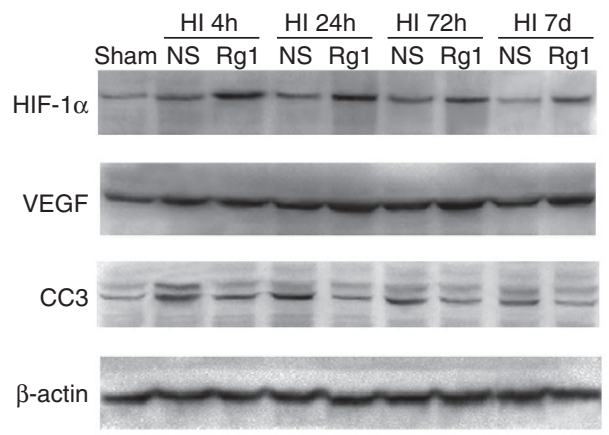

C

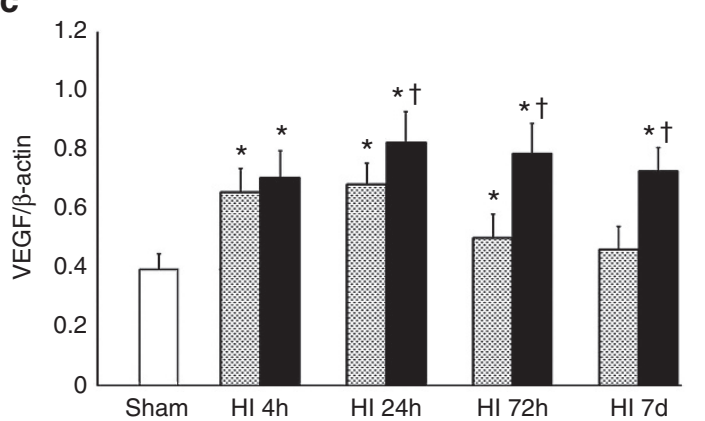

b

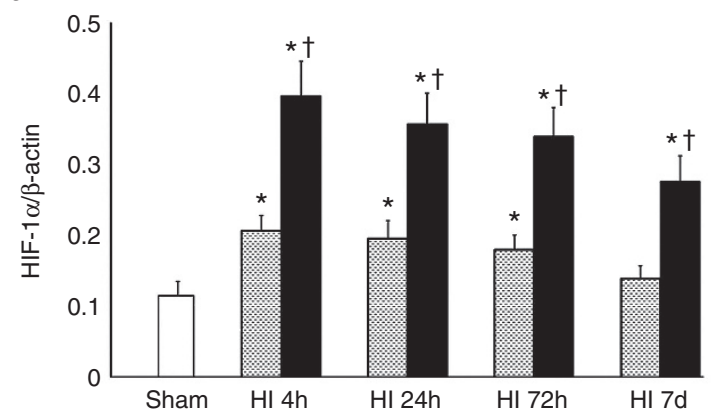

d

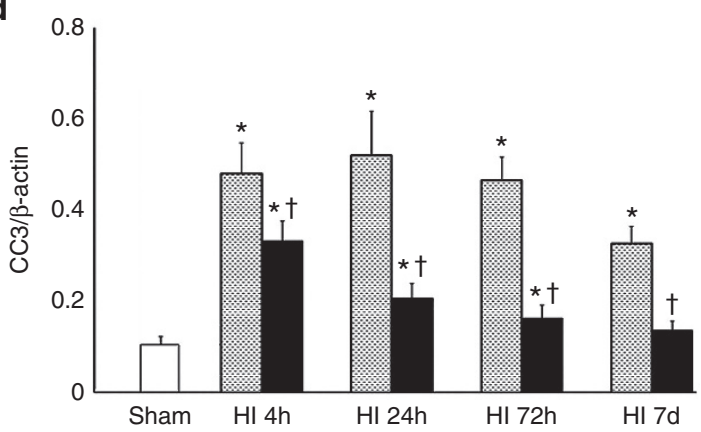

Figure 5. Western blot quantitative analysis of HIF-1 $\alpha$, VEGF, and CC3 protein expression (a) and integrated density values were shown in b-d. (a) Western blot was conducted using cortex and hippocampus tissues of right brain to examine HIF-1 $\alpha$, VEGF, and CC3 protein expression in sham controls and at $4 \mathrm{~h}$, $24 \mathrm{~h}, 72 \mathrm{~h}$, and $7 \mathrm{~d}$ after $\mathrm{HI}$ in rats received NS or Rg1treatment. (b) After normalization with $\beta$-actin, we found that HIF-1 $\alpha$ expression was induced after $\mathrm{HI}$ (approximately a 1.80 -fold at $4 \mathrm{~h}$, a 1.70 -fold at $24 \mathrm{~h}$ and a 1.56 -fold at $72 \mathrm{~h}$, compared with sham controls, $P<0.05$ ) and restored to a low level similar to sham at $7 \mathrm{~d}$ (approximately a 1.21-fold, $P>0.05$ ). Rats received Rg1 showed a higher and longer-lasting expression of HIF- $1 \alpha$ than that received only $\mathrm{HI}$ treatment at each time point (approximately a 1.92-fold at $4 \mathrm{~h}$, a 1.82 -fold at $24 \mathrm{~h}$, a 1.89 -fold at $72 \mathrm{~h}$ and a 1.98 -fold at $7 \mathrm{~d}$ after $\mathrm{HI}, P<0.05$ ). (c) Analogue to HIF-1 $\alpha$, compared to sham controls, VEGF was also obviously induced after $\mathrm{HI}$ (approximately a 1.67 -fold at $4 \mathrm{~h}$, a 1.74 -fold at $24 \mathrm{~h}$ and a 1.28 -fold at $72 \mathrm{~h}, P<0.05$ ), and then declined at $7 \mathrm{~d}$ (approximately a 1.18-fold, $P>0.05$ ), and Rg1 facilitated maintenance of a higher expression level of VEGF (approximately a 1.21-fold at $24 \mathrm{~h}$, a 1.56 -fold at $72 \mathrm{~h}$ and a 1.57 -fold at $7 \mathrm{~d}$, compared with rats received only $\mathrm{HI}$ treatment, $P<0.05$ ). (d) Meanwhile, we found obvious activation of caspase-3 protein (in form of CC3) after $\mathrm{HI}$ stress (approximately a 4.60 -fold at $4 \mathrm{~h}$, a 4.98 -fold at $24 \mathrm{~h}$, a 4.45 -fold at $72 \mathrm{~h}$ and a 3.13 -fold at $7 \mathrm{~d}$ after $\mathrm{Hl}$, compared with sham controls, $P<0.05)$, the expression level of CC3 that received Rg1 was significantly lower than that received NS at $4 \mathrm{~h}(31.8 \%$ lower), $24 \mathrm{~h}(60.2 \%$ lower), $72 \mathrm{~h}\left(65.0 \%\right.$ lower), and $7 \mathrm{~d}\left(58.5 \%\right.$ lower) after HI. Histograms: white fill $=$ sham, spots fill $=\mathrm{HI}$, black fill $=\mathrm{HI}+\mathrm{Rg} 1 .{ }^{*} P<0.05$ compared with sham control; $+P<0.05$ compared with $\mathrm{HI}$ group at the same time. $n=6$ for each time point at each group.

blot showed CC3 expression was consistent with neuronal AI detected by TUNEL: it was low in sham controls, upregulated after HI, and decreased after intervention with Rg1. In contrast, HI-stimulated HIF-1 $\alpha$ and VEGF expressions were stronger and maintained a high expression level for a longer time in Rg1-treated pups than those in NS-treated ones. These findings suggested that antiapoptotic effect of $\mathrm{Rg} 1$ may be related to HIF-1 $\alpha$-mediated inactivation of caspase- 3 and transcriptional activation of VEGF, an important neuroprotective factor.

It is well known that HIF-1 $\alpha$ is induced following tissue hypoxia/ischemia and then promotes transcription of VEGF, which is crucial for neo-vascularization $(12,13)$.Early studies have suggested that Rg1 is a potent stimulator of VEGF and exerts angiogenic effects in vitro $(9,27)$ and in vivo $(10,28)$. In present study, we also found Rg1 promoted in vivo vasculogenesis following HI and HIF-1 $\alpha /$ VEGF expression was increased in this process, furthermore it should be noticed that HIF$1 \alpha$ expression peaked at $4 \mathrm{~h}$, earlier than that seen in VEGF expression (which peaked at $24 \mathrm{~h}$ following $\mathrm{HI}$ ), suggesting the involvement of HIF-1 $\alpha /$ VEGF signaling cascade. All these findings provide evidence that the Rg1-associated angiogenesis in brain repair after HI may be HIF-1 $\alpha$ /VEGF-dependent.
In summary, we found in present study that compare to GM1, Rg1 showed multiple beneficial potential in antiapoptosis and angiogenesis in brain repair following neonatal HI. HIF-1 $\alpha /$ VEGF is plausibly neural-protective and may represent an endogenous adaptive response of the developing rat brain to HI. HIF-1 $\alpha$, VEGF and CC3 signaling cascade may represent a critical component in neuronal survival and angiogenesis, and further investigations using inhibitors of HIF- $1 \alpha$ and VEGF are needed to directly clarify this signaling cascade. Rg1 could facilitate the inhibition of caspase- 3 activation and subsequent neuronal apoptosis, as well as neo-vascularization by strengthening HIF-1 $\alpha$ /VEGF signaling pathway. In view of the above findings, it was proposed the therapeutic properties of Rg1 in neonates with HI brain injury.

\section{METHODS}

Neonatal HI Brain Injury Rat Model and Drug Administration All animal researches were approved by the Sichuan Academy of Medical Sciences Committee on Animal Research. SD rats $10 \mathrm{~d}$ after birth (P10) weighing 17-21 g, without gender selection, were used in this study. Rat pups were assigned to four different groups that subjected to four different treatments: HI group $(n=27)$ : an HI model was established with a modified RVM as previously described (22): each pup was anesthetized with ethyl ether, the right common carotid 
artery was separated and permanently ligated with surgical silk through a midline cervical incision. After recovering with the dam for $0.5 \mathrm{~h}$, pups were exposed to hypoxic inhalation (8\% oxygen and $92 \%$ nitrogen) for $2.5 \mathrm{~h}$. A constant temperature of $37^{\circ} \mathrm{C}$ was maintained throughout the entire procedure. Sham group $(n=27)$ : pups underwent anesthesia and the right common carotid artery was only separated, without ligation or subsequent hypoxic inhalation. HI+Rg1 group $(n=27): 40 \mathrm{mg} / \mathrm{kg} \mathrm{Rg} 1$ dissolved in $0.1 \mathrm{ml} \mathrm{NS}$ was intraperitoneally injected immediately after $\mathrm{HI}$ and such injection was repeated every $24 \mathrm{~h}$ for $7 \mathrm{~d}$. HI+GM1 group $(n=27): 40 \mathrm{mg} / \mathrm{kg}$ GM1 was intraperitoneally injected as the same as that in Rg1 group. Intraperitoneal injection of $0.1 \mathrm{ml} \mathrm{NS}$ was performed in HI group or sham group, in the same way as that of in Rg1 and GM1 group. Behavior activity levels were monitored at 4, 24, $72 \mathrm{~h}$ and $7 \mathrm{~d}$ after HI, pups in sham group served as controls. 3 groups of sham-operated rats separately treated with NS, $40 \mathrm{mg} / \mathrm{kg}$ Rg1 or $40 \mathrm{mg} / \mathrm{kg} \mathrm{GM1}$ every $24 \mathrm{~h}$ for $7 \mathrm{~d}$ (sham, sham+ Rg1, sham + GM1, respectively) were also included to evaluate toxicity of Rg1 or GM1 on neuronal viability in the immature brain.

\section{Histology and Immunohistochemistry}

Paraffin-embedded sections of the right hemisphere were used for hematoxylin and eosin (HE) histological staining and immunohistochemisty, as described previously $(10,22)$. Briefly, right cerebral hemisphere was fixed with $4 \%$ paraformaldehyde and coronally cut into 5 - $\mu \mathrm{m}$-thick sections, and then sections were stained with HE staining or used for IHC. For immunohistochemistry, paraffin sections were processed for heat-based antigen retrieval and endogenous peroxidase was inhibited with $0.3 \%$ hydrogen peroxide in methanol. Goat serum (1:10) served as the blocking agent before incubation with rabbit antiHIF- $1 \alpha$ antibody (1:50, Santa Cruz, Dallas, TX), rabbit anti-CC3 antibody (1:200, CST, Danvers, MA), rabbit anti-VEGF antibody (1:200, Santa Cruz), and rabbit anti-von willebrand factor (vwf) antibody (1:200, Millipore, Darmstadt, Germany). After incubation overnight at $4^{\circ} \mathrm{C}$, biotin-labeled secondary antibody work solution (ZSGB-BIO, Beijing, China) was loaded, followed by freshly prepared ABC (avidin biotin complex, ZSGB-BIO), and then 3-3' diaminobenzidine (DAB, KPL, Gaithersburg, MD) was used as a chromogen. Finally, sections were counter-stained with hematoxylin.

\section{TUNEL Labeling}

Two coronally slices cut from right hemisphere of each rat were used for examination of apoptotic cell death using an In Situ Cell Death Detection Kit (Roche, Basel, Switzerland) according to the manufacturer's protocol $(7,22)$. Paraffin-embedded sections were used for terminal deoxynucleotidyl transferase-mediated deoxyuridine triphosphate-biotin nick end labeling (TUNEL). Sections were deparaffinized, rehydrated, and treated with $0.3 \%$ hydrogen peroxide as described above. Sections were incubated at $37^{\circ} \mathrm{C}$ for $20 \mathrm{~min}$ with proteinase $\mathrm{K}(20 \mu \mathrm{g} / \mathrm{ml})$ and then incubated at $37^{\circ} \mathrm{C}$ for $1 \mathrm{~h}$ with biotinylated nucleotide and the terminal deoxynucleotidyl transferase, recombinant (rTdT) enzyme. After the washes, sections were incubated with converter-POD at $37^{\circ} \mathrm{C}$ for $30 \mathrm{~min}$ and then detected with DAB. Three fields in cortex and hippocampus of each slice were chosen randomly at $400 \times$ magnifications to count apoptotic cells and total cells. The apoptotic index (AI) was calculated as follows: $\mathrm{AI}=($ number of apoptotic cells/total number counted $) \times 100 \%$.

\section{Western Blot Analysis}

Tissue lysates were generated from the cortex and hippocampus of right cerebral hemisphere and quantified as described previously (29). An equal amount of protein was separated on $8 \%$ SDS polyacrylamide gels (for HIF- $1 \alpha$ and VEGF) or $12 \%$ SDS polyacrylamide gels (for CC3), transferred to PVDF membranes (Roche), and blocked in 5\% bovine serum albumin in TBS containing $0.1 \%$ Tween 20 . The membranes were incubated for $1 \mathrm{~h}$ at room temperature, and then overnight at $4{ }^{\circ} \mathrm{C}$, with one of primary antibodies as described in IHC part: rabbit HIF- $1 \alpha$ polyclonal antibody (1:200), rabbit anti-CC3 polyclonal antibody $(1: 1,000)$, rabbit anti-VEGF polyclonal antibody $(1: 1,000)$, and mouse anti- $\beta$-actin monoclonal antibody (1:3,000, Sigma). Following washes, membranes were incubated with HRP-conjugated goat anti-rabbit IgG or goat anti-mouse secondary antibody $(1: 3,000$, ZSGB-BIO). The signals of bound antibodies were identified with an enhanced chemiluminescence kit (ECL, Amersham, Pittsburgh,PA).
The integrated density value of each band was assessed using a Gelpro image analyzer (Bio-Rad, Hercules, CA), and protein levels were normalized to $\beta$-actin as a loading control.

\section{Statistical Analysis}

All data are representative of at least three experiments per condition. Data are presented as mean \pm SD. Significances were calculated with a one-way ANOVA and a LSD post hoc test. For comparison of the Longa grades in the different groups the Kruskal-Wallis one-way ANOVA on ranks and post hoc pairwise multiple comparison procedures were used. $P$ values were calculated with the software SPSS (IBM, Armonk, NY) and considered significant when $P<0.05$.

\section{ACKNOWLEDGMENTS}

We thank Dezhi Mu and Yi Qu for their theoretical and technical assistance.

\section{STATEMENT OF FINANCIAL SUPPORT}

This work was supported by grants from Doctoral Foundation of Sichuan Academy of Medical Sciences \& Sichuan Provincial People's Hospital (No. 30305030580 to B.T.) and Technology Development Fund of Chengdu University of Traditional Chinese Medicine (No. 030029052 to D.W.).

Disclosure: The authors have no conflicts of interest to declare.

\section{REFERENCES}

1. Gill MB, Perez-Polo JR. Hypoxia ischemia-mediated cell death in neonatal rat brain. Neurochem Res 2008;33:2379-89.

2. Wu CC, Chen YC, Chang YC, et al. Human umbilical vein endothelial cells protect against hypoxic-ischemic damage in neonatal brain via stromal cell-derived factor $1 / \mathrm{C}-\mathrm{X}-\mathrm{C}$ chemokine receptor type 4 . Stroke 2013;44:1402-9.

3. Lin M, Sun W, Gong W, Ding Y, Zhuang Y, Hou Q. Ginsenoside Rg1 protects against transient focal cerebral ischemic injury and suppresses its systemic metabolic changes in cerabral injury rats. Acta Pharm Sin B 2015;5:277-84.

4. Deng Y, Zhang T, Teng F, et al. Ginsenoside Rg1 and Rb1, in combination with salvianolic acid B, play different roles in myocardial infarction in rats. J Chin Med Assoc 2015;78:114-20.

5. Shen L, Zhang J. Ginsenoside Rg1 increases ischemia-induced cell proliferation and survival in the dentate gyrus of adult gerbils. Neurosci Lett 2003;344:1-4.

6. He Q, Sun J, Wang Q, Wang W, He B. Neuroprotective effects of ginsenoside Rg1 against oxygen-glucose deprivation in cultured hippocampal neurons. J Chin Med Assoc 2014;77:142-9.

7. Wang D, Huang Y, Li Q, Xu S, Liu X. [Anti-apoptotic effect of ginsenoside Rg1 on neuron after neonatal hypoxia ischemia brain damage]. Zhongguo Xiu Fu Chong Jian Wai Ke Za Zhi 2010;24:1107-12.

8. Miao HH, Zhen Y, Ding GN, Hong FX, Xie ZC, Tian M. Ginsenoside Rg1 attenuates isoflurane-induced caspase-3 activation via inhibiting mitochondrial dysfunction. Biomed Environ Sci 2015;28:116-26.

9. Shi AW, Wang XB, Lu FX, Zhu MM, Kong XQ, Cao KJ. Ginsenoside Rg1 promotes endothelial progenitor cell migration and proliferation. Acta Pharmacol Sin 2009;30:299-306.

10. Wang DJ, Li QY, Xu SJ, Zeng N. [Effect of ginsenoside Rg1 on angiogenesis after neonatal hypoxia ischemia brain damage in rats]. Sichuan Da Xue Xue Bao Yi Xue Ban 2011;42:503-7.

11. Tang BZ, Zhao FY, Qu Y, Mu DZ. [Hypoxia-inducible factor-1alpha: a promising target for tumor therapy]. Ai Zheng 2009;28:775-82.

12. Tang B, Qu Y, Wang D, Mu D. Targeting hypoxia inducible factor-1 $\alpha$ : a novel mechanism of ginsenoside $\operatorname{Rg} 1$ for brain repair after hypoxia/ischemia brain damage. CNS Neurol Disord Drug Targets 2011;10:235-8.

13. Tang B, Qu Y, Mu D. [Research progress of hypoxia inducible factor lalpha in hypoxic-ischemic injury]. Zhongguo Xiu Fu Chong Jian Wai Ke Za Zhi 2009;23:755-8.

14. Tang B, Qu Y, Zhao F, et al. In vitro effects of hypoxia-inducible factor 1alpha on the biological characteristics of the SiHa uterine cervix cancer cell line. Int J Gynecol Cancer 2009;19:898-904.

15. Wu J, Pan Z, Wang Z, et al. Ginsenoside Rg1 protection against $\beta$-amyloid peptide-induced neuronal apoptosis via estrogen receptor $\alpha$ and 


\section{Articles | Tangetal.}

glucocorticoid receptor-dependent anti-protein nitration pathway. Neuropharmacology 2012;63:349-61.

16. Yang $\mathrm{N}$, Chen $\mathrm{P}$, Tao Z, et al. Beneficial effects of ginsenoside-Rg1 on ischemia-induced angiogenesis in diabetic mice. Acta Biochim Biophys Sin (Shanghai) 2012;44:999-1005.

17. Liu G, Wang T, Wang T, Song J, Zhou Z. Effects of apoptosis-related proteins caspase-3, Bax and Bcl-2 on cerebral ischemia rats. Biomed Rep 2013;1:861-7.

18. Jiang B, Xiong Z, Yang J, et al. Antidepressant-like effects of ginsenoside Rg1 are due to activation of the BDNF signalling pathway and neurogenesis in the hippocampus. Br J Pharmacol 2012;166:1872-87.

19. Li L, Tian J, Long MK, et al. Protection against experimental stroke by ganglioside GM1 is associated with the inhibition of autophagy. PLoS One 2016;11:e0144219.

20. Aureli M, Mauri L, Ciampa MG, et al. GM1 ganglioside: past studies and future potential. Mol Neurobiol 2016;53:1824-42.

21. Sakurai M, Morita T, Takeuchi T, Shimada A. Relationship of angiogenesis and microglial activation to seizure-induced neuronal death in the cerebral cortex of Shetland Sheepdogs with familial epilepsy. Am J Vet Res 2013;74:763-70.

22. Li L, Qu Y, Li J, Xiong Y, Mao M, Mu D. Relationship between HIF-1alpha expression and neuronal apoptosis in neonatal rats with hypoxia-ischemia brain injury. Brain Res 2007;1180:133-9.
23. Zachary I. Neuroprotective role of vascular endothelial growth factor: signalling mechanisms, biological function, and therapeutic potential. Neurosignals 2005;14:207-21.

24. Lahiani A, Zahavi E, Netzer N, et al. Human placental eXpanded (PLX) mesenchymal-like adherent stromal cells confer neuroprotection to nerve growth factor (NGF)-differentiated PC12 cells exposed to ischemia by secretion of IL-6 and VEGF. Biochim Biophys Acta 2015;1853:422-30.

25. Khan M, Dhammu TS, Matsuda F, et al. Promoting endothelial function by S-nitrosoglutathione through the HIF-1 $\alpha /$ VEGF pathway stimulates neurorepair and functional recovery following experimental stroke in rats. Drug Des Devel Ther 2015;9:2233-47.

26. Bhuiyan MI, Kim JC, Hwang SN, Lee MY, Kim SY. Ischemic tolerance is associated with VEGF-C and VEGFR-3 signaling in the mouse hippocampus. Neuroscience 2015;290:90-102.

27. Leung KW, Ng HM, Tang MK, Wong CC, Wong RN, Wong AS. Ginsenoside-Rg1 mediates a hypoxia-independent upregulation of hypoxia-inducible factor-1 $\alpha$ to promote angiogenesis. Angiogenesis 2011;14:515-22.

28. Zhang YJ, Zhang XL, Li MH, et al. The ginsenoside Rg1 prevents transverse aortic constriction-induced left ventricular hypertrophy and cardiac dysfunction by inhibiting fibrosis and enhancing angiogenesis. J Cardiovasc Pharmacol 2013;62:50-7.

29. Zhang L, Qu Y, Tang B, et al. Integrin $\beta 8$ signaling in neonatal hypoxicischemic brain injury. Neurotox Res 2012;22:280-91. 